\title{
ULTRAVIOLET COLORS OF SUBDWARF O STARS
}

\author{
Paul R. Wesselius \\ Kapteyn Institute
}

\section{INTRODUCTION AND INSTRUMENT}

The group of subdwarf 0 stars consisting of field stars and some central stars of old planetary nebulae does occupy an intresting place in the HR diagram. Greenstein and Sargent (1974) have tried to establish this place, and conclude that especially the hottest ones need ultraviolet data to improve the values of effective temperature and absolute luminosity.

I therefore observed some twenty sdo stars in the far ultraviolet using the spectrophotometer in the Netherlands' satellite ANS (van Duinen et al, 1975). It has five quite rectangular photometric bands at 1550 (50 or 150), 1800 (150), 2200 (200), 2500 (150), and 3300 (100) $\AA$. The numbers between brackets indicate the widths of the bands in Angstroms. These measurements could be done with the ANS-UV instrument mainly because of its small field of view ( $2.5 \times 2.5$ arc minute), a factor of more than 10 better than the aperture of comparable previous satellite instruments: The background light is much suppressed and close-by stars can be avoided.

\section{DATA REDUCTION}

Most objects have been observed several times. Usually the counting rates of a single observation (photomultipliers in the photon counting mode have been used) have an error (one standard deviation) of typically a few percent. No variability was detected at this level for any of the sdo stars, and therefore all observations of an object were averaged together. The reduction procedure leading to "net" count rates at a specific date, and by 


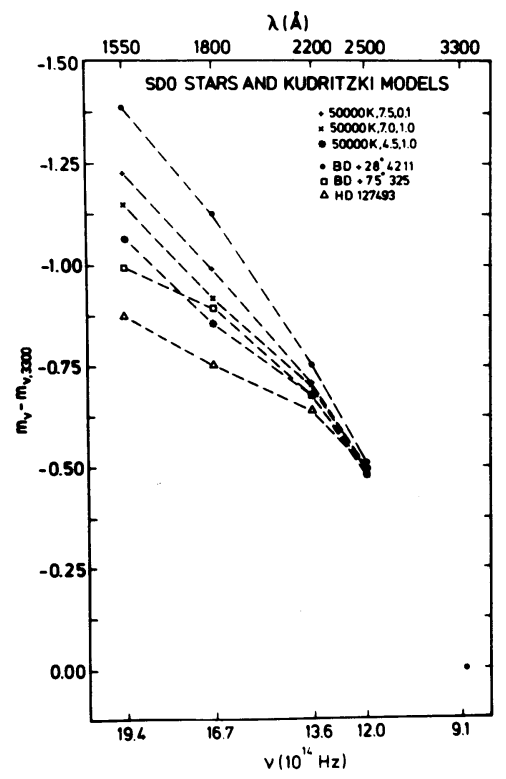

Fig. I

For the models the numbers behind the plotting symbols indicate effective temperature, log $\mathrm{g}$, and He abundance. The three sdO stars were corrected for $\mathrm{E}(\mathrm{B}-\mathrm{V})=0.03$, 0.03 and 0.07 from top to bottom.

applying an absolute calibration to absolute fluxes and magnitudes, has been described by van Duinen et al (1977).

The extinction correction poses a special problem for these stars. The extinction bump in the UV at $2200 \AA$ (one of the ANS-UV channels has been chosen at precisely this wavelength) makes it possible for most hot objects to correct for extinction by "smoothing" the bump (see e.g. Pottasch et al, 1977). The hot sdo stars however, can have quite high helium abundances, and consequently the absorption edge due to He II $(n=3)$ at $2050 \AA$ may show up. This would produce a "bump" at $2200 \AA$ going the other way. An extensive investigation was undertaken and I believe that the problem has been solved satisfactorily. This will be discussed in a future paper.

\section{COMPARISON WITH MODELS; DISCUSSION}

Dr. Kudritzki kindly folded the UV fluxes of some of his sdo models (as described in his paper of 1976) with the ANS passbands. A few models are shown in Fig. 1 along with the data on three subdwarf 0 stars. The He edge should be (see the models) and is (HD 127493) apparent in the ANS-UV data. The steep rise towards $1550 \AA$ exhibited by the models and the data (BD + 280 4211) is caused by the high gravities, and is in sharp contrast to the more gentle rise of the normal 0 stars. Fig. 2 shows 18 sdo stars with some main-sequence stars from 07 to $\mathrm{B} 4$; it is evident that the sdo stars are a lot more "ultraviolet" than the mainsequence stars. 


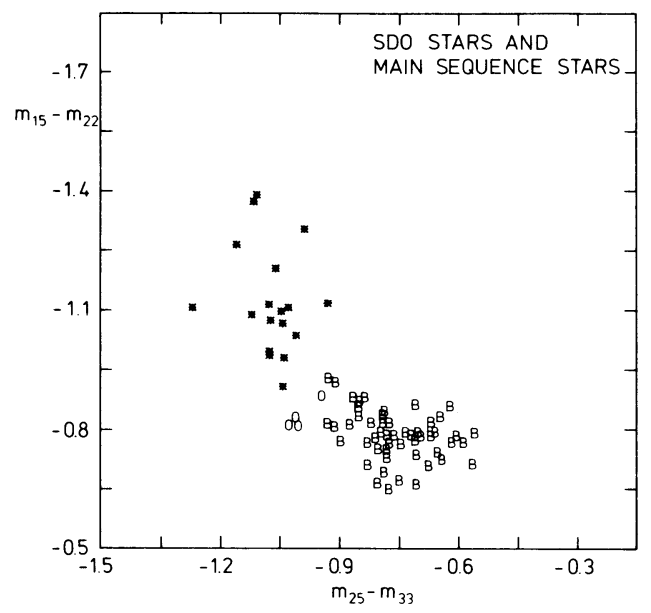

\section{Fig. 2}

Color-color plot; the stars have been corrected for interstellar extinction. 15, 22, 25 and 33 indicate the 1550, 2200, 2500 and $3300 \AA$ band. The "star" symbols represent sdO stars, "O" and "B" refer to spectral classes $O$ and $B$.

\section{REFERENCES}

Greenstein, J.L. and Sargent, A.I. (1974). Astrophys. J. Suppl. $28,157$.

Kudritzki, R.P. (1976). Astron. Astrophys. 52, 11.

Pottasch, S.R., Wesselius, P.R., Wu, C.C. and van Duinen, R.J. (1974). Astron. Astrophys. 54, 435.

van Duinen, R.J., Aalders, J.W.G., Wesselius, P.R., Wildeman, K.J., Wu, C.C., Luinge, W. and Snel, D. (1975). Astron. Astrophys. 39, 159.

van Duinen, R.J., Wesselius, P.R., Wu, C.C., Aalders, J.W.G. and Kester, D. (1977). in preparation. 


\section{DISCUSSION}

SHIPMAN: What is the helium abundance of the high-helium star you mentioned ( $B D$ - 310 4800) determined from the optical spectrum? Are there any hydrogen lines visible in its spectrum?

WESSELIUS: As far as I know this star shows very strong helium lines and no Balmer lines.

STENCEL: The Kudritzki models have a large range of gravities ( $\log \mathrm{g}=4.5$ to 7.5 ) yet do not match the observed flux range. Do you require a larger range of gravities to succeed here?

WESSELIUS: No, a larger range of effect temperatures is needed. NORRIS: I would like to comment that your results concerning the temperatures and helium abundances of the sdo stars appear to support the suggestion originating, I think, with Strittmatter that there exists a boundary in the (log $\mathrm{g}$, Teff) plane hotter than which surface peculiarities, in particular helium weakness, cannot exist. According to Strittmatter this boundary is defined by the condition that mass loss removes an atmosphere on a timescale short compared to that necessary for diffusive processes to be operative. If one accepts a sequence of evolution from sdO to DA, and that sdO stars are not helium weak, then your results support the hypothesis that as the stars become cooler diffusion turns on as the stars cross the 'Strittmatter boundary' and diffusive timescales become shorter than those for mass loss. Would you comment further on the high helium abundances in the sdo stars, and on these ideas?

WESSELIUS: sdO stars are defined as showing He II $4686 \AA$, and all that we have anlyzed so far show large He abundances.

NORRIS: Perhaps Dr. Greenstein or Dr. Shipman could comment.

GREENSTEIN: I believe that not all subdwarf 0 stars need show He II. Very hot degenerate stars have no helium at the surface; hot sdO's (a) have He II, if there are many lines including ionized metals, or (b) show almost no lines, except shallow hydrogen. The hottest star in the ANS diagram, +2804211 , has no or very weak $\mathrm{He}$ II $4686 \AA$, yet is an 0 subdwarf. The fainter nuclei of planetary nebulae have weak He II. The description of type 0 by the presence of $\mathrm{He}$ II is near breaking down. The settling out of helium may occur as log $\mathrm{g}$ increases, but in addition the composition may be affected by details at the end of the planetary-nucleus stage.

WESSELIUS: Among the sdO stars I've observed there are three nuclei of planetary nebulae, and they have rather strong helium lines, and furthermore look completely similar to the other sdo stars.

WALBORN: I think the spectra of subdwarf 0 stars show He II lines by definition. Certainly +2804211 has very strong $4686 \AA$ line and +750325 has strong $4200,4541 \AA$ lines as well.

GREENSTEIN: Not necessarily in high-gravity stars, which may also be helium-deficient at their surfaces. 